\title{
In vitro Antibacterial Activity of Organic Extracts of Aloe barbadensis against Multi-drug Resistant Pseudomonas aeruginosa Isolated from Wound Specimens
}

\author{
Mamata Adhikari ${ }^{1}$, Anil Kumar Sah' ${ }^{2}$ Dev Raj Joshi ${ }^{1 *}$ \\ ${ }^{1}$ Central Department of Microbiology, Tribhuvan University, Kirtipur, Kathmandu, Nepal \\ ${ }^{2}$ Annapurna Neurological Institute and Allied Science Hospital, Maitighar, Kathmandu, Nepal, Maitighar, \\ Kathmandu
}

*Corresponding author: Dr. Dev Raj Joshi, Central Department of Microbiology, Tribhuvan University, Kathmandu, Nepal; E-mail: dev.joshi@microbiotu.edu.np

\begin{abstract}
Objectives: In order to investigate alternate therapeutic option, this study was carried out to assess the in vitro antibacterial activity of gel extract of Aloe barbadensis against multiple antibiotic resistant Pseudomonas aeruginosa isolated from wound specimens.
\end{abstract}

Methods: A total of 180 different wound specimens collected in a hospital, were subjected to isolate and identify $P$. aeruginosa by cultural methods. Antibiotic susceptibility testing was done by KirbyBauer disc diffusion method to screen multidrug resistant isolates. A. barbadensis extracts were prepared using aqueous and organic solvents and their in vitro inhibitory action was evaluated by agar well diffusion methods.

Results: Out of total, 38 (21.1\%) of the wound specimens showed the occurrence of $P$. aeruginosa, among which 15 (39\%) isolates were multi-drug resistant. Organic extracts of various concentrations $(0.2-0.8 \mathrm{v} / \mathrm{v} \%)$ inhibited $66.7 \%$ of MDR and all non-MDR $(\mathrm{n}=23)$ P. aeruginosa with zone of inhibition ranging from $9.5 \pm 1.0$ to $21.3 \pm 2.2 \mathrm{~mm}$ but not by aqueous extract. A positive Pearson's correlation ( $\mathrm{r}=0.983$ ) was found between antibacterial effect and concentrations of the extracts. The antibacterial activity of organic extracts was statistically associated with antibiotic resistance profile of the organism $(\mathrm{p}<0.05)$.

Conclusion: Organic extracts of $A$. barbadensis revealed variable in vitro inhibitory action against both MDR and non-MDR P. aeruginosa isolated from wound specimens. Although further confirmation is needed, aloe gel extract may be applied as an alternate treatment option.

Key words: Pseudomonas aeruginosa, MDR, Aloe barbadensis, organic extracts, antibacterial activity, wound infection

\section{INTRODUCTION}

There is an increasing incidence of Pseudomonas aeruginosa in wound infections (Masaadeh and Jaran 2009) because an open wound provides a moist, warm and nutritious environment perfect for microbial colonization and proliferation (Benbow 2010). P. aeruginosa is an opportunistic and highly resistant nosocomial pathogen. It can grow on a wide range of substrates and quickly respond to environmental alteration (Lambert 2002). It can express virulence factors and surface proteins affecting wound healing.

Date of Submission: November 2, 2018

Published Online: January 2019
The development of a wound infection following elective surgery or traumatic injury remains a major cause of morbidity in patients (Kim et al. 1999).

Several studies have shown the emergence of multidrug resistant (MDR) isolates of $P$. aeruginosa associated with community acquired and nosocomial infections (Bhandari et al. 2015; Chaudhary et al. 2016; Fan et al. 2016). Ali et al. (2015) observed higher resistance of $P$. aeruginosa to the commonly used antibiotics like ofloxacin $(61.3 \%)$, cefepime $(57.3 \%)$, ceftazidime $(53.9 \%)$ and amikacin (53.0\%). It is obvious that the

Date of Acceptance: December 5, 2018

DOI: https:/ / doi.org/10.3126/tujm.v5i0.22315 
widespread MDR P. aeruginosa threaten the life of the infected patients globally.

The search of newer antibacterial sources has been fostered because antibiotic alternatives may reduce the use of antibiotics. In other hand, $80 \%$ of the population in developing countries mainly relies on traditional herbal medicines for therapies against infections (WHO 2002) due to wide availability, easy use, low cost, minimal or no adverse effect on human health. During the past several decades, both in vivo and in vitro studies have demonstrated higher antibacterial potency of biologically active compounds extracted from medicinal plants (Malterud et al. 1993; Goudarzi et al.2015). Aloe barbadensis a medicinal plant, contains over 75 potentially active constituents including vitamins, enzymes, minerals, sugars, lignin, saponins, lupeol, salicylic acids, amino cinnamonic acid, phenols and sulfur (Surjushe et al. 2008). Many of these compounds are bactericidal, virucidal, fungicidal and antiinflammatory in nature (Hamman 2008). Furthermore, the inhibitory action of extracts of $A$. barbasensis against many gram negative pathogens including $P$. aeruginosa has been recognized (Ibrahim et al. 2011; Renisheya et al. 2012; , Abakar et al. 2017). However, its antibacterial activity against MDR $P$. aeruginosa has rarely been evaluated in Nepal.

Therefore, in this study, we tested in vitro inhibitory action of different organic extracts of locally available A. barbadensis at different concentrations against MDR and non-MDR $P$. aeruginosa isolated from various wound specimens.
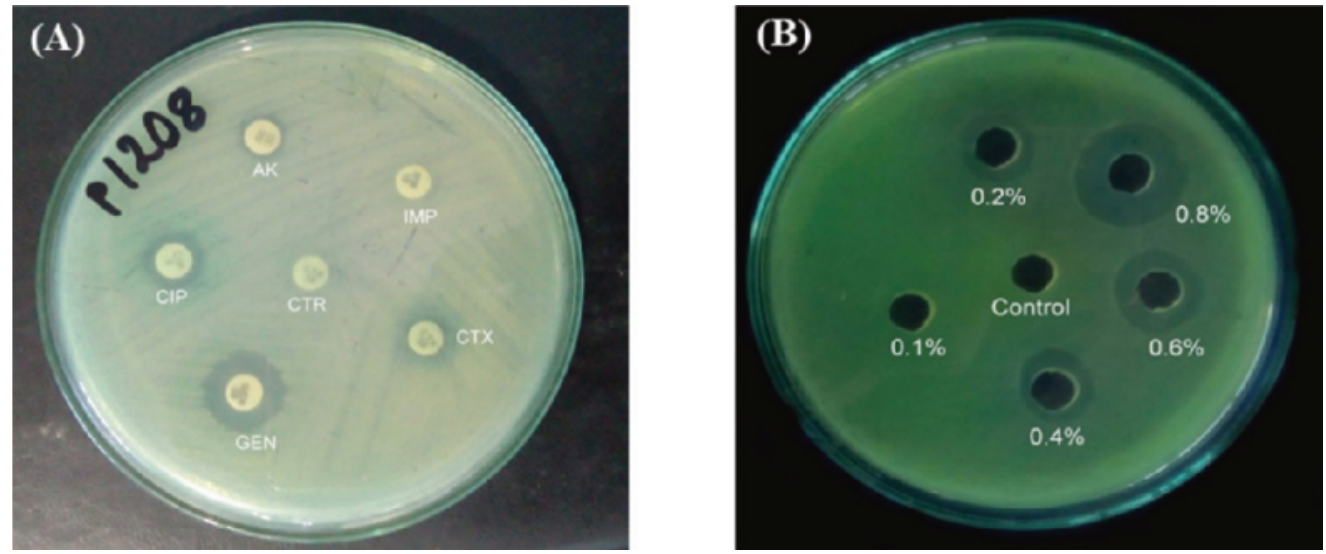

Figure 2: P. aeruginosa (P/1208) on Muller Hinton Agar plates showing antibiotic sensitivity testing (A) and antibacterial activity by DMSO extract of A. barbadensis (B). Abbreviations for antibiotics are as mentioned in the text. For in vitro antibacterial activity testing, $0 \%$ (control) to $0.8 \%(\mathrm{v} / \mathrm{v})$ DMSO extracts was used. Zone of inhibitions were measured as $\mathrm{mm}$. 
Preparation of A. barbadensis gel extracts: Mature, healthy and fresh leaves of $A$. barbadensis were collected and washed in the running tap water for 10 minutes, rinsed with sterile distilled water and surface sterilized with $70 \%$ ethyl alcohol followed by $0.1 \% \mathrm{HgCl}_{2}$. The leaves were dissected longitudinally and the colorless parenchymatous tissue (aloe gel) was scraped out using a sterile knife. Fresh aloe gel was dried at $80^{\circ} \mathrm{C}$ for 48 hours and then powdered. Ten grams of the powder was soaked in $100 \mathrm{ml}$ of four different solvents (distilled water, DMSO, acetone and ethanol) separately for 24 hours. The content was filtered through Whatman filter paper (except for DMSO extract) and filtrate was evaporated to dryness. The dried powdered extract was dissolved in distilled water for further use. In case of acetone extract, extracted powder was dissolved in $\mathrm{NaOH}$ and neutralized with $0.15 \mathrm{M} \mathrm{HCl}$.

Determination of antibacterial activity of $A$. barbadensis gel extract: The antibacterial activity of $A$. barbadensis gel extract against the $P$. aeruginosa isolates was tested by agar well diffusion technique (Figure1B). Each of aloe extract was diluted with distilled water to prepare test solutions of $0.1 \%, 0.2 \%, 0.4 \%, 0.6 \%$ and $0.8 \%(\mathrm{v} / \mathrm{v})$ concentrations. Exactly $100 \mu \mathrm{L}$ of each test solution of each of aloe gel extracts were loaded into each of the $5 \mathrm{~mm}$ diameter wells on Mueller Hinton agar which were swabbed with an overnight broth culture of the organism and incubated at $37{ }^{\circ} \mathrm{C}$ for 24 hours. The respective pure solvent (acetone, ethanol and DMSO) was used as the control. In vitro inhibitory action was measured in terms of zone of inhibition $(\mathrm{mm})$ around the test wells.

\section{RESULTS}

$P$. aeruginosa isolates from wound specimens and antibiotic resistance: Out of total 180 wound specimens, $38(21.1 \%)$ of the specimens showed the occurrence of $P$. aeruginosa. The highest numbers of the $P$. aeruginosa isolates were recovered from burn wound infections ( $\mathrm{n}=7,35.0 \%$ ) while the lowest number from pus specimens $(n=9,13.9 \%)$ (Table 1$)$.

Table 1: Distribution of $P$. aeruginosa in various wound specimens

\begin{tabular}{lccc}
\hline Type of specimen & Total & Growth obtained $($ P. aeruginosa) & Percent \\
\hline Burn wound swabs & 20 & 7 & 35.0 \\
Postoperative wound swabs & 40 & 8 & 20.0 \\
Chronic wound swabs & 55 & 14 & 25.5 \\
Pus specimens & 65 & 9 & 13.9 \\
\hline Total & $\mathbf{1 8 0}$ & $\mathbf{3 8}$ & $\mathbf{2 1 . 1}$ \\
\hline
\end{tabular}

Antibiotic susceptibility testing revealed that out of total 38 isolates of $P$. aeruginosa, 15 (39.0\%) isolates were designated as MDR P. aeruginosa (Figure 2) while others were regarded as non-MDR. The highest number

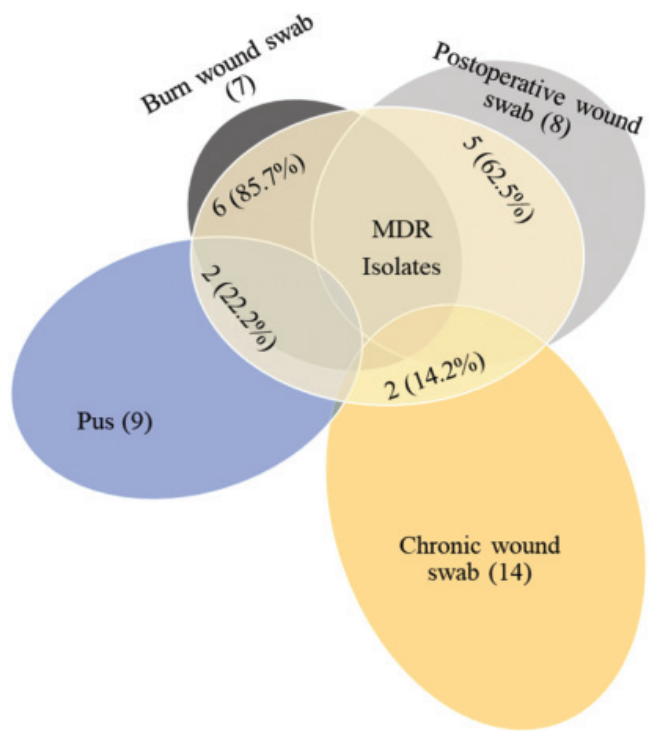

of MDR P. aeruginosa were isolated from burn wound specimens $(n=6,85.7 \%)$ followed by postoperative wound $(n=5,62.5 \%)$.

Figure 2: Venn diagram showing distribution of multidrug resistant (MDR) $P$. auroginosa isolates in different clinical samples. Parentheses in each sample represent the number of total $P$. aeruginosa isolates $(\mathrm{N}=38)$. Frequencies of MDR isolates $(n=15)$ from each sample are expressed as number $(n / N$ $\%)$. 
In vitro antibacterial activity of $A$. barbadensis extracts against $P$. aeruginosa: In order to evaluate the inhibitory potential of $A$. barbadensis against wound infecting $P$. aeruginosa, both the MDR ( $\mathrm{n}=15)$ and non-MDR $(\mathrm{n}=23)$ isolates were tested. Production of clear inhibition zone around theagarwell containing theextract was considered as 'sensitive' isolate, otherwise 'resistant' as no such zone was observed. While aqueous extract did not show any antibacterial effect, extracts with all three organic solvents; acetone, ethanol and DMSO demonstrated antibacterial effect at varying concentrations.

As demonstrated in Figure 3, among total of 38 isolates of $P$. aeruginosa, 33 (86.8\%) were inhibited by at least one of organic extract of $A$. barbadensis. Out of the total 15 MDR isolates, $10(66.7 \%)$ were found to be sensitive to aloe extracts. Interestingly, all $23(100 \%)$ non- MDR isolates of $P$. aeruginosa were found to be sensitive to aloe extracts.

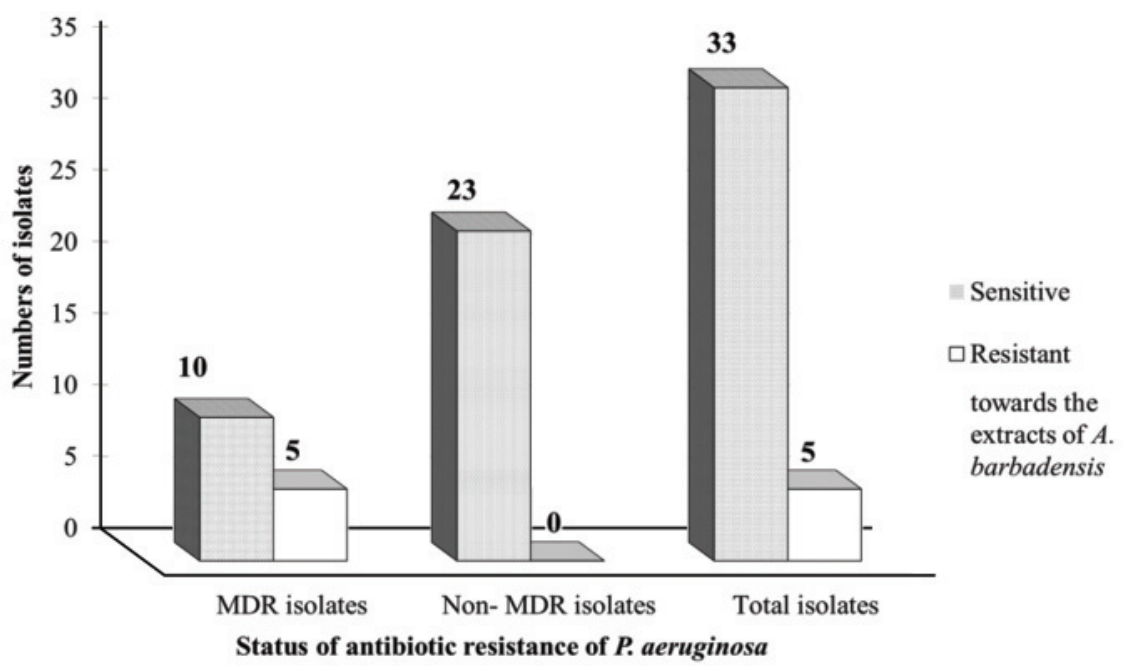

Figure 3: Bar diagram showing antibacterial activity of organic extracts of $A$. barbadensis against MDR and non MDR isolates of $P$. aeruginosa. The numbers represent the frequencies of isolates either sensitive or resistant towards at least one organic extract of $A$. barbadensis.

The maximum zone of inhibition of $21.3 \pm 2.2 \mathrm{~mm}$ was revealed by acetone extract at concentration $0.8 \%$ against the MDR isolate of $P$. aeruginosa obtained from a burn wound while minimum zone of inhibition of $9.5 \pm 1.0 \mathrm{~mm}$ was shown by DMSO extract at $0.2 \%$ concentration. DMSO extract of A. barbadensis showed increasing size of zone of inhibition as concentration was raised from $0.2 \%$ to $0.8 \%$ (Pearson's correlation coefficient, $r=0.9951$ ) against MDR isolates of $P$. aeruginosa and the linear curve was well fitted with $\mathrm{R}^{2}$ of 0.9999 (Figure 4A). Comparatively, acetone extracts revealed highest in vitro inhibitory activity (larger zone of inhibition in all concentrations). Similar to the results against MDR isolates, non-MDR $P$. aeruginosa were also inhibited by 0.4 to $0.8 \%$ concentrations of acetone and DMSO extracts and $0.6-0.8 \%$ concentrations of ethanol extracts (Figure 4B). The results showed comparatively similar pattern of antibacterial activity of organic extracts of $A$. barbadensis against both the MDR and non-MDR isolates.

Association between multidrug resistance and antibacterial activity of $A$. barbadensis: Despite the similar inhibitory pattern of individual aloe extracts, we attempted to determine the association between MDR status of $P$. aeruginosa and gross sensitivity towards $A$. barbadensis applying chi-square test. 


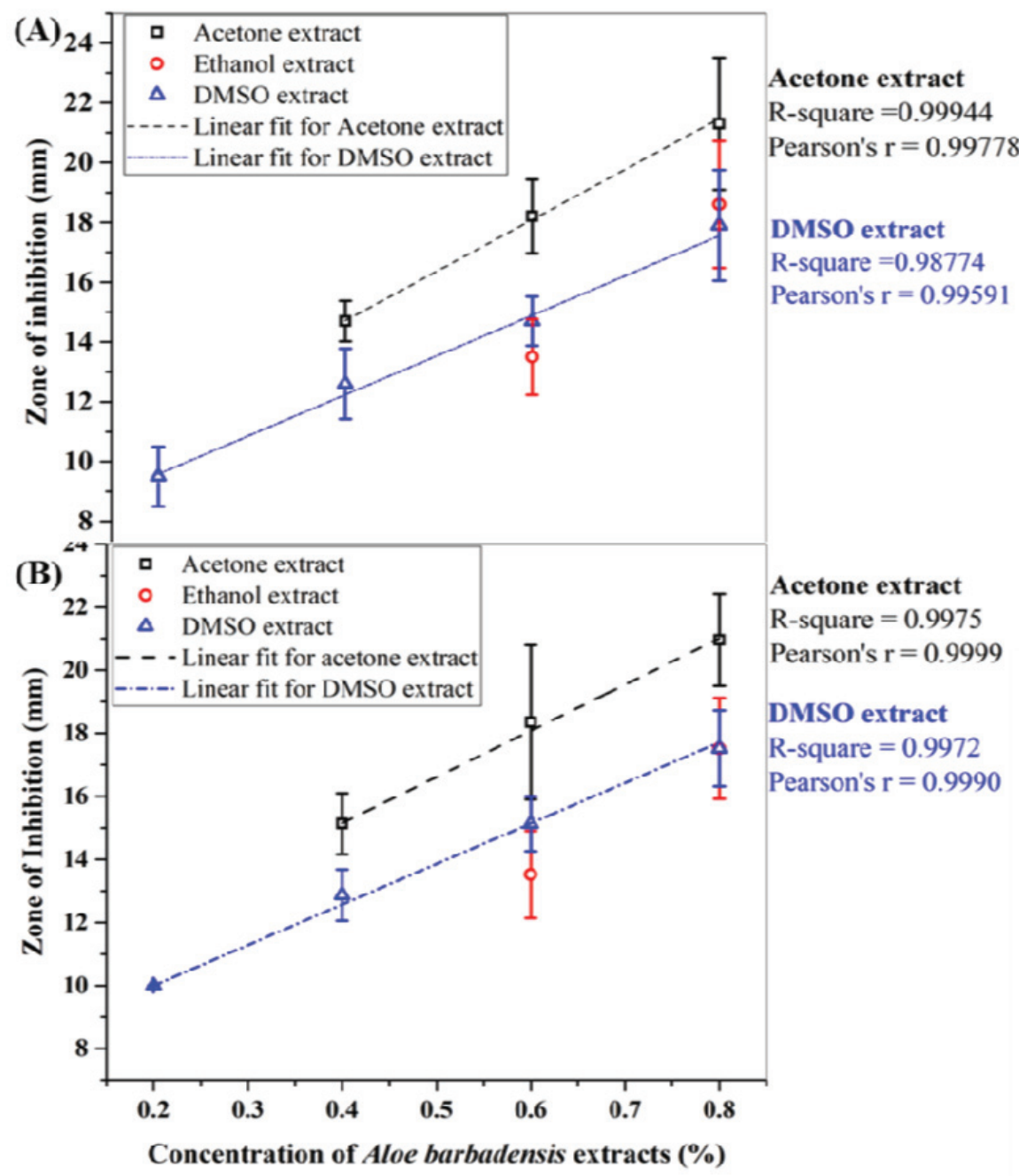

Figure 4: Antibacterial activity of Aloe barbadensis extracts at different concentration against multidrug resistant (A) and non-multidrug resistant (B) isolates of $P$. aeruginosa. Error bars represent the standard deviation. Pearson's $r$ is correlation coefficient between concentration of extracts and ZOI produced by respective extracts. Since ethanol extract showed inhibition only at two concentrations, statistical analysis was not valid.

Compared to all non-MDR isolates, only two-third of MDR isolates $(\mathrm{n}=5)$ of $P$. aeruginosa were sensitive towards Aloe extracts. A significant statistical

Table 2: Sensitivity of MDR isolates to A. barbadensis association $(\mathrm{p}<0.05)$ was revealed between MDR status and the sensitivity towards $A$. barbadensis (Table 2).

\begin{tabular}{lcccc}
\hline \multirow{2}{*}{ Status of antibiotic resistance } & \multicolumn{2}{c}{ Sensitivity to $\boldsymbol{A}$. barbadensis extract } & \multirow{2}{*}{ Total (\%) } & \multirow{2}{*}{ p-value* } \\
\cline { 2 - 3 } & Sensitive (\%) & Resistant (\%) & $15(100.0)$ & 0.006 \\
\hline MDR & $10(66.7)$ & $5(33.3)$ & $23(100.0)$ & \\
Non- MDR & $23(100.0)$ & $5(0.00)$ & $38(100.0)$ & \\
Total & $33(86.8)$ & $5(13.2)$ & & \\
\hline
\end{tabular}

${ }^{*}$ Chi-square test

In order to understand which organic solvent extracts of $A$. barbadensis produce maximum inhibitory activity against $P$. aeruginosa isolates, the sizes of zone of inhibition $(\mathrm{mm})$ revealed by each organic extracts at different concentrations were compared for MDR and non-MDR isolates. Though acetone extracts at all concentrations $(0.4-0.8 \%)$ revealed higher magnitude of inhibition than DMSO and ethanol extracts, there was no significant difference in diameter of the zone of inhibition produced by different extracts for MDR and non-MDR isolates ( $p>0.05)$ for all concentrations (Table 3). 
Table 3: Association between drug resistance and antibacterial activity of organic extracts of $A$. barbadensis at different concentrations

\begin{tabular}{lccc}
\hline Extract & Concentration & Diameter of zone of inhibition (mm) against & Non-MDR \\
\hline Acetone & & MDR & $21.0 \pm 1.5$ \\
Ethanol & $0.8 \%$ & $21.3 \pm 2.2$ & $17.5 \pm 1.6$ \\
DMSO & & $18.6 \pm 2.1$ & $17.5 \pm 1.2$ \\
\hline Acetone & & $17.9 \pm 1.9$ & $18.4 \pm 2.4$ \\
Ethanol & $0.6 \%$ & $18.2 \pm 1.2$ & $13.5 \pm 1.4$ \\
DMSO & & $13.5 \pm 1.3$ & $15.1 \pm 0.9$ \\
\hline Acetone & & $14.7 \pm 0.8$ & $15.1 \pm 1.0$ \\
Ethanol & $0.4 \%$ & $14.7 \pm 0.7$ & 0 \\
DMSO & & 0 & $12.9 \pm 0.8$ \\
\hline Acetone & & $12.6 \pm 1.2$ & 0 \\
Ethanol & $0.2 \%$ & 0 & 0 \\
DMSO & & $9.5 \pm 1.0$ & 0.191 \\
\hline
\end{tabular}

*ANOVA

\section{DISCUSSION}

In order to investigate an alternate therapeutic option, this study was carried out to assess the antibacterial activity of $A$. barbadensis against MDR $P$. aeruginosa isolated from wound specimens. Since $P$. aeruginosa is generally a part of wound infection etiology, the target pathogen was isolated from different types of wound specimens. In this study, $P$. aeruginosa was recovered from $21.1 \%$ as key etiological agent of wound infections. However, previous studies reported the prevalence of $P$. aeruginosa as $5.6 \%$ to $44.0 \%$ (Bhatt and Lakhey 2007; Bangera et al. 2015; Rai et al. 2017). Considering variable prevalence of $P$. aeruginosa in different types of wound infections (Rajbahak et al. 2012; Bastola et al. 2017; Serra et al. 2015; Kshetry et al. 2015), we selected burn wound swab, postoperative wound swab, chronic wound swab and pus specimens for this study where the burn wound specimens contributed maximum of $P$. aeruginosa isolates (35\%).

Infections caused by $P$. aeruginosa are often severe and life threatening (Ahmed et al. 2008) and are difficult to treat because of its limited susceptibility to antimicrobial agents and the high frequency of the emergence of antibiotic resistance during therapy (Niederman, 2001; Paladino et al. 2002). Because we found high prevalence of MDR P. aeruginosa in wound infections $(39.5 \%)$ which is higher than the study of Bangera et al. (2015) with resistance against the most of aminoglycoside, fluoroquinolone and beta-lactams (Rajbahak et al. 2012), we argue that there may be increasing trend of antibiotic resistance of $P$. aeruginosa causing wound infection. The high resistance of $P$. aeruginosa may be due to the intrinsic resistance like chromosomally encoded $\beta$-lactamase, biofilm formation, energy dependent efflux (Hancock 1997) and acquired resistance by mutational events leading to over-expression of endogenous $\beta$-lactamases or efflux pumps, diminished expression of specific porins and target site modifications. Acquired resistance is also due to acquisition of resistance genes which mainly refers to transferable $\beta$-lactamases and aminoglycosidemodifying enzymes.

In order to assess the in vitro inhibitory activity of $A$. barbadensis, we analyzed if there is relation among concentration, types of extraction solvents and antibacterial activity of the $A$. barbadensis. We found that organic extracts with acetone, ethanol and DMSO extracts gave zone of inhibition in certain concentrations but not by aqueous extract. It may be due to partial solubility or insolubility of active compound in aqueous solution (non-polar nature and active compound). Broadly, the magnitude of inhibition was obtained in the pattern of acetone > DMSO > ethanol extracts $(0.4-0.8 \%)$. The results of this study corroborated with Ibrahim et al. (2011) that reported better antimicrobial activity of the acetone extract as compared to ethanol and aqueous extracts. In this study, the maximum zone of inhibition $(21.3 \pm$ $2.2 \mathrm{~mm}$ ) by acetone extract at $0.8 \%$ concentration was found which was to be similar ( $21 \mathrm{~mm}$ by acetone extract) to the study of Abakar et al. (2017) for P. aeruginosa suggesting that acetone could be the best organic solvent for extraction. Various extracts showed different zone of inhibition which may be attributed to different solubility of various compounds found in Aloe barbadensis. More polar substances extract more of the compounds embedded within the plant cells 
(Karpagam and Devaraj 2011). Our experiment showed that, the inhibitory action increases proportionately with the increment of concentration of the extracts corroborating with results of Renisheya et al. (2012).

Among MDR isolates, two-thirds of the isolates were sensitive to aloe extract while all non-MDR isolates were found sensitive. The antibacterial activity of extract was found statistically associated with antibiotic resistance profile of the organism $(\mathrm{p}<0.05)$. This indicates the multidrug resistant $P$. aeruginosa were less susceptible to aloe extract. Irrespective of the different wound specimens, the zone of inhibition was nearly similar for all isolates of $P$. aeruginosa. However, in a particular concentration of all extracts, there was no significant difference in diameter of the zone of inhibition for MDR and non- MDR isolates ( $p$ $>0.05)$.

From this study it is clear that $A$. barbadensis demonstrates inhibitory activity against $P$. aeruginosa indicating its therapeutic potential. Lawrence et al. (2009) identified the compounds with maximum antibacterial activity against $P$. aeruginosa as p-coumaric acid, pyrocatechol and cinnamic acid. Pyrocatechol denatures proteins and disrupts cell membranes (Cowan 1999). Cinnamic acid inhibits glucose uptake and ATP production in the resting cells of bacteria (Kouassi and Shelef 1998). Similarly, p-coumaric compound is reported to increase the lag phase of the microorganism (Baranowski et al. 1980) and is also able to inhibit the enzymatic activity of the microorganisms (Weir et al. 2004). However, in this study the active compounds were not identified. So it is recommended for further research that isolation of key bioactive compound of Nepalese variety of $A$. barbadensis would be useful asset in this regard.

\section{CONCLUSION}

Both the MDR and non-MDR isolates of $P$. aeruginosa recovered from wound specimens were inhibited in vitro by organic extracts of A. barbadensis. However, comparison study showed slightly higher frequency of non-MDR isolates was sensitive towards organic extracts of A. barbadensis. Acetone extract had comparatively better inhibitory activity and magnitude of in vitro inhibition was positively correlated with concentration of extracts. Though further confirmation is necessary, in the light of the multiple antibiotic resistance problems, this study showed that $A$. barbadensis may be used as an alternate therapeutic option for the treatment of Pseudomonas caused infections more particularly wound infections.

\section{ACKNOWLEDGEMENTS}

The authors acknowledge University Grants Commission, Nepal for the partial financial support and Annapurna Neurological Institute and Allied Science Hospital, Nepal for laboratory facilities.

\section{CONFLICT OF INTEREST}

The authors declare no conflict of interest.

\section{REFERENCES}

Abakar HOM, Bakhiet SEA and Abadi RSM (2017). Antimicrobial activity and minimum inhibitory concentration of Aloe vera sap and leaves using different extracts. J Pharmacogn Phytochem 6(3): 297-303.

Ahmed BED, Magda EN, Rawia B and Amr ES (2008). Pseudomonas aeruginosa exotoxin A: its role in burn wound infection and wound healing. Egypt J Plast Reconstruct Surg 32: 59-65.

Ali Z, Mumtaz N, Naz SA, Jabeen N and Shafique M (2015). Multi-drug resistant Pseudomonas aeruginosa: a threat of nosocomial infections in tertiary care hospitals. J Pak Med 65(1): 12-16.

Bangera D, Shenoy SM and Saldanha DRM (2015). Clinico-microbiological study of Pseudomonas aeruginosa in wound infections and the detection of metallo- $\beta$-lactamase production. Int Wound J 13: 1-4.

Baranowski JD, Davidson PM, Nagel CW and Branen AL (1980). Inhibition of Saccharomyces cerevisiae by naturally occurring hydroxycinnamates. J. Food Sci 45: 592-594.

Bastola R, Parajuli P, Neupane A and Paudel A (2017). Surgical site infections: distribution studies of sample, outcome and antimicrobial susceptibility testing. J Med Microb Diagn 6(1): 1-7.

Benbow M (2010). Wound swabs and chronic wounds. Practice Nurse 39(9): 27-30.

Bhandari P, Thapa G, Pokhrel BM, Bhatta DR and Devkota U (2015). Nosocomial isolates and their resistant pattern in ICU patients at National Institute of Neurological and Allied Sciences, Nepal. Int J Microbiol (572163): 1-6.

Bhatt CP and Lakhey M (2008) The distribution of pathogens causing wound infection and their antibiotic susceptibility pattern. J Nepal Health Res Counc 5: 22-26.

Chaudhary AK, Bhandari D, Amatya J, Chaudhary P and Achrya B (2016). Metallo $\beta$-lactamase producing gram-negative bacteria among patients visiting Shahid Gangalal National Heart Centre. Austin J Microbio Res 2: 208-212.

Clinical and Laboratory Standards Institute (CLSI) (2016). Performance standards for antimicrobial 
susceptibility testing; $26^{\text {th }}$ informational supplement. CLSI document M100-S26. Wayne, PA: Clinical and Laboratory Standards Institute.

Cowan MM (1999). Plant products as antimicrobial agents. Clin Microbiol Rev 12(4): 564-582.

Fan X, Wu Y, Xu M, Xu ZP, Kudinha T, Bazaj A, Kong F and Xu YC (2016). Diverse genetic background of multi-drug resistant Pseudomonas aeruginosa from mainland China, and emergence of an extensively drug-resistant ST292 clone in Kunming. Sci Rep 6: $1-8$.

Goudarzi M, Fazeli M, Azad M, Seyedjavadi SS and Mousavi R (2015). Aloe vera gel: effective therapeutic agent against multidrug-resistant Pseudomonas aeruginosa isolates recovered from burn wound infections. Chemother Res Practice 2015 (639806): 1-5.

Hamman JH (2008). Composition and application of Aloe vera leaf gel. Molecules 13: 1599-1616.

Hancock REW (1997). The bacterial outer membrane as a drug barrier. Trends Microbiol 5: 37-42.

Ibrahim M, Srinivas M and Lakshmi Narasu M (2011). Phyto-chemical analysis and antimicrobial evaluation of Aloe vera gel against some human and plant pathogens. Asian J Curr Chem 1(1): 1-11.

Karpagam T and Devaraj AR (2011). Studies on the efiicacy of Aloe vera on antimicrobial activity. IJRAP 2(4): 1286-1289.

Kim HS, Kacew S and Lee BM (1999). In vitro chemopreventive effect of plant polysaccharides (Aloe barbadensis Miller, Lentinus edodes, Gandoderm lucidum, and Coriolus vesicolor). Carcinogenesis 20: 1637-1640.

Kouassi Y and Shelef, LA (1998). Inhibition of Lysteria monocytogenes by cinnamic acid: possible interaction of the acid with cysteinyl residues. J Food Safety 18(3): 231-242.

Kshetry AO, Lekhak B and Raghubanshi BR (2015). Antibiogram of bacteria isolated from wound exudates. Int J Biol Med Res 6(2): 4997-5002.

Lambert PA (2002). Mechanisms of antibiotic resistance patterns of gram negative isolates in a tertiary care hospital of Nepal. Asian J Pharma Clin Res 7: 30-33.
Lawrence R, TripathiP and JeyakumarE(2009). Isolation, purification and evaluation of antibacterial agents from Aloe vera. Braz J Microbiol 40: 906-915.

Malterud KE, Farbrot TL and Huse AE (1993). Antioxidant and radical scavenging effects of anthraquinones and anthrones. Pharmacology 47: 77-85.

Masaadeh HA and Jaran AS (2009). Incident of Pseudomonas aeruginosa in post- operative wound infection. Am J Infect Dis 5(1): 1-6.

Niederman MS (2001). Impact of antibiotic resistance on clinical outcomes and the cost of care. Crit Care Med 29: 114-120.

Paladino JA, Sunderlin JL, Price CS and Schentag JJ (2002). Economic consequences of antimicrobial resistance. Surg Infect (Larchmont) 3: 259-267.

Rai S, Yadav UN, Pant ND, Yakha JK, Tripathi PP, Poudel A and Lekhak B (2017). Bacteriological profile and antimicrobial susceptibility patterns of bacteria isolated from pus/wound swab samples from children attending a tertiary care hospital in Kathmandu, Nepal. Int J Microbiol 2017 (2529085): 1-5.

Rajbahak S, Shrestha C, Shrestha J and Singh A (2014). Bacteriological changes of burn wounds with time and their antibiogram. Sci Rep 12: 70-76.

Renisheya JJMT, Johnson M, Nancy BS, Laju RS, Anupriya G and Renola JJET (2012). Anti-bacterial and antifungal activity of Aloe vera gel extract. IJBAR 3: 184-187.

Serra R, Grande R, Butrico L, Rossi A, Settimio UF, Caroleo B, Amato B, Gallelli L and de Franciscis $S$ (2015). Chronic wound infections: the role of Pseudomonas aeruginosa and Staphylococcus aureus. Expert Rev Anti Infect Ther 13(5): 605-613.

Surjushe A, Vasani R and Saple DG (2008). Aloe vera: a short review. Indian J Dermatol 53: 163-166.

Weir TL, Park SW and Vivanco, JM (2004). Biochemical and physiological mechanisms mediated by allelochemicals. Curr Opinion Plant Biol 7: 472479 .

World Health Organization (WHO) (2002). Traditional medicine strategy (2002-2005). WHO/EDM/ TRM/2002.1. Geneva, Switzerland: World Health Organization. 